
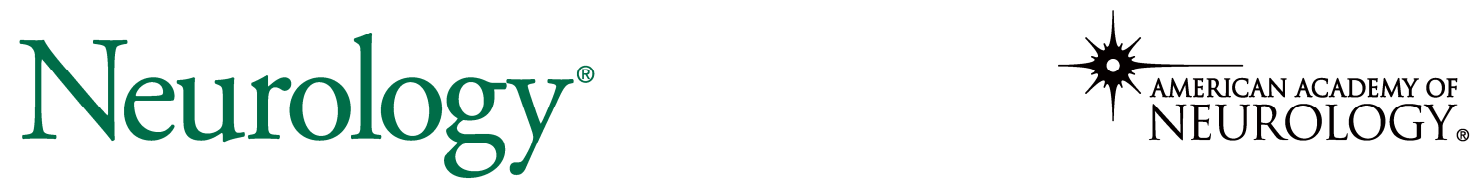

The most widely read and highly cited peer-reviewed neurology journal The Official Journal of the American Academy of Neurology

Neurology Publish Ahead of Print

DOI: 10.1212/WNL.0000000000012663

\title{
Clinical Reasoning: A 55-Year-Old Man With Odd Behavior and Abnormal Movements
}

\section{Author(s):}

Paul McIntosh, MD ${ }^{1}$; Burton Scott, PhD, MD ${ }^{1}$

Neurology® Published Ahead of Print articles have been peer reviewed and accepted for publication. This manuscript will be published in its final form after copyediting, page composition, and review of proofs. Errors that could affect the content may be corrected during these processes. 


\section{Corresponding Author:}

Paul McIntosh

paul_mcintosh@outlook.com

Affiliation Information for All Authors: 1. Duke University Medical Center

\section{Contributions:}

Paul McIntosh: Drafting/revision of the manuscript for content, including medical writing for content; Study concept or design

Burton Scott: Drafting/revision of the manuscript for content, including medical writing for content

Number of characters in title: 78

Abstract Word count:

Word count of main text: 1470

References: 9

Figures: 1

Tables: 0

Search Terms: [ 161 ] All Movement Disorders, [ 164 ] Huntington's disease, [ 170 ] Chorea

Study Funding: The authors report no targeted funding

Disclosures: Paul McIntosh reports no disclosures related to this work. Burton Scott reports no disclosures related to this work.

\section{$\underline{\text { Section } 1}$}

A 55-year-old man with an extensive psychiatric history that included PTSD, anxiety, depression, and schizophrenia treated with quetiapine and trazodone was admitted to the hospital from an assisted living facility (ALF) for subacute on chronic failure to thrive and concern for psychosis, with recent worsening ongoing for 6 weeks. He was recently discharged from an outside psychiatric facility, and at the ALF he demonstrated odd behavior such as eating food off the floor and spitting on staff in addition to persistent poor intake of fluids, food, and medications due to issues with regurgitation and dysphagia. He had been evaluated by 
gastroenterology for over a decade for these issues in addition to chronically elevated aspartate aminotransferase (AST) and alanine aminotransferase (ALT). An extensive workup including CT imaging, esophagogastroduodenoscopy, esophagram, motility study, and a gastric emptying study was largely unremarkable. In the setting of his psychiatric history and reported history of frequently spitting out food, there was concern for a volitional component to his regurgitation.

Prior to hospitalization, he had been seen by outpatient neurologists for hyperkinetic movements and was given the diagnosis of Tourette's syndrome at age 40. A diagnosis of akathisia was entertained as he had been on quetiapine for psychiatric symptoms for many years.

During this admission, the neurological exam was notable for a thin male with frequent hyperkinetic movements affecting the trunk and bilateral upper extremities with a random and flowing quality, most prominent when sitting up. He denied experiencing an urge to move and he could not suppress the movements. There was a mild milk-maid's grip and he was unable to maintain the tongue fully protruded for 5 seconds. Otherwise his exam demonstrated normal extraocular movements, intact sensation to multiple modalities, and no overt appendicular ataxia. Deep tendon reflexes were difficult to assess as he would not cooperate with the examination. He was able to sit unassisted and walk unassisted, although he exhibited a staggering gait and his tandem gait was unsteady. He denied having a family history of similar symptoms. Lab workup was notable for a creatinine of $1.6 \mathrm{mg} / \mathrm{dL}$ (baseline $1.2 \mathrm{mg} / \mathrm{dL}$ ) and elevated AST (304 U/L) and $\operatorname{ALT}(154 \mathrm{U} / \mathrm{L})$.

\section{Questions for consideration:}

1. What type of hyperkinetic movement disorder does this patient demonstrate?

2. What is on the differential based on history and exam? 


\section{$\underline{\text { Section } 2}$}

The patient was unable to perform sustained motor activity as demonstrated by a milkmaid's hand grip (on squeezing the examiner's fingers, the patient exhibited waxing-waning grip strength as if milking the examiners fingers) and difficulty with sustained tongue protrusion. These features of motor impersistence are characteristic of chorea as were the random limb movements that appeared to flow from one body part to another. ${ }^{1}$

His prior diagnosis of a tic disorder and possible akathisia were not consistent with his symptoms, as he denied experiencing an urge to move and he could not suppress the movements. In addition, tics should consist of repetitive and stereotyped motor (or phonic) behaviors whereas his movements were random and irregular. Furthermore, the diagnosis of Tourette's requires a history of vocal and motor tics beginning prior to age $18 .^{2}$

The differential diagnosis for chorea includes structural disorders affecting the basal ganglia or subthalamic nucleus (vascular insults, tumors, demyelinating lesions), toxic-metabolic derangements (nonketotic hyperglycemia, electrolyte derangements, carbon monoxide poisoning), drug-induced (dopaminergic therapy, stimulants, anticonvulsants, tardive syndrome), infectious (toxoplasmosis, HIV encephalopathy, prion disease), systemic autoimmune processes (lupus, antiphospholipid syndrome, Sydenham chorea), hereditary (Huntington disease, neuroacanthocytosis, brain iron accumulation disorders, Wilson disease), and paraneoplastic syndromes (anti-CRMP-5, anti-NMDA, among others) in addition to other causes (polycythemia vera, chorea gravidarum, postpump chorea, and psychogenic). ${ }^{1}$

The temporal course of chorea can assist in narrowing down the differential diagnosis. Acquired or sporadic causes of chorea tend to present acutely to subacutely, whereas hereditary causes tend to present more insidiously. The anatomic distribution of chorea can also assist in the 
differential diagnosis, as unilateral chorea tends to occur with structural causes, nonketotic hyperglycemia, and oftentimes antiphospholipid syndrome. ${ }^{1}$ Thus, based on this patient's gradual-onset history and generalized chorea, a hereditary cause was suspected.

\section{Question for consideration:}

1. What diagnostic studies would you recommend?

\section{$\underline{\text { Section } 3}$}

The patient had neurological, psychiatric, and liver abnormalities, raising concern for Wilson disease. Wilson disease has several abnormal movements associated with the condition, including tremor and ataxia. Chorea can be present, although it is not a typical manifestation. ${ }^{3} \mathrm{~A}$ serum copper and ceruloplasmin was recommended. These tests were both in the normal range.

Due to his chronic transaminitis (elevated AST and ALT), an extensive gastroenterological and rheumatological workup was performed but was unrevealing. As the transaminitis could indicate muscle breakdown as well as liver pathology, a creatine kinase (CK) level was checked and was found to be elevated at $4753 \mathrm{IU} / \mathrm{L}$. From chart review of outside records, his baseline CK ranged from 600-800 IU/L. His CK returned to his prior elevated baseline with the administration of intravenous fluids.

MRI brain with and without contrast was recommended given his longstanding abnormal movements to assess for a structural etiology or evidence of a neurodegenerative process. This revealed atrophy of the bilateral caudate (Figure 1).

\section{Questions for consideration:}

1. Based on the results of the serum studies and neuroimaging, what more targeted workup should be conducted to establish a diagnosis? 


\section{$\underline{\text { Section } 4}$}

The differential for choreiform movements, psychiatric history, feeding issues, and caudate atrophy on brain MRI includes Huntington disease and Huntington-like disorders, in addition to neuroacanthocytosis. Elevated CK levels are expected for neuroacanthocytosis, as there is an associated myopathy which may be subclinical. ${ }^{4}$ To further investigate these diagnostic possibilities, genetic testing for Huntington's was sent and a blood smear was requested to assess for acanthocytosis. The Huntington disease genetic testing returned with a normal number of CAG (cytosine-adenine-guanine) repeats. On the blood smear, there were $\sim 20 \%$ acanthocytes per hematology review. A diagnosis of neuroacanthocytosis was made.

\section{$\underline{\text { Discussion }}$}

Neuroacanthocytosis syndromes are characterized by acanthocytes on blood smear (red cells with "spikes") in addition to the presence of chorea, feeding dystonia, lip and tongue biting, tics, and neuropsychiatric symptoms. Notably the neuropsychiatric features can precede overt motor features, causing diagnostic confusion with a tardive syndrome. Neuropathy, myopathy, cardiomyopathy, seizures, and parkinsonism may also occur. ${ }^{1}$

Core subsets of neuroacanthocytosis include the autosomal recessive chorea-acanthocytosis (ChAc) caused by mutations in the VPS113A gene and the X-linked Mcleod syndrome (MLS) caused by mutations in the $X K$ gene encoding the Kell antigen on erythrocytes. ChAc has a mean age of onset of 35 years and tends to be associated with severe feeding dystonia and subsequent weight loss, difficulties with saccades, a "rubber man" posture and gait, and atrophy of the caudate nucleus. ${ }^{1,4,5}$ The feeding dystonia is described as uncontrollable tongue protrusion out of the mouth while eating, often resulting in biting the tongue and dropping food from the mouth. ${ }^{7}$ The "rubber man" appearance results from truncal instability and sudden, violent trunk spasms in 
these patients. ${ }^{8}$ MLS has a mean age of onset of 45 years and a higher risk of cardiomyopathy manifesting as atrial fibrillation, other arrhythmias, or dilated cardiomyopathy. ${ }^{1,4,5}$ Both ChAc and MLS may have chronic hyperCKemia. ${ }^{5}$ Rhabdomyolysis is a rare complication, as was present in this patient. ${ }^{5,9}$ Recognition of MLS is of particular importance since patients can experience life-threatening blood transfusion reactions to allogeneic blood transfusion. ${ }^{1}$

It is important to note that apart from the core neuroacanthocytosis syndromes, there are other degenerative movement disorders where acanthocytosis is occasionally seen. These include pantothenate kinase-associated neurodegeneration (PKAN) and Huntington-disease-like 2 (HDL2). PKAN is an autosomal recessive disorder that tends to present in childhood with dystonia as opposed to chorea, and has a characteristic MRI signature called the "eye of the tiger sign" on T2 weighted imaging due to brain iron deposition, identified as T2 hyperintensity of the center of the globus pallidus interna surrounded by hypointensity. ${ }^{4}$ HDL2 is an autosomal dominant disorder that is similar to both ChAc and MLS in that it is a phenocopy of Huntington disease, often presenting in young adulthood. It is most commonly seen in individuals of African descent. It is distinct from ChAc and MLS in that there are no muscle abnormalities and CK levels are normal. ${ }^{5,7}$ C9orf72 repeat expansion is another cause of hereditary chorea and is now recognized as the most common phenocopy of HD. ${ }^{1}$ However, acanthocytosis has not been documented in $\mathrm{C} 9$ orf72 disease.

He and his family received genetic counseling with respect to the diagnosis of neuroacanthocytosis. Symptomatic management with tetrabenazine $12.5 \mathrm{mg}$ daily for choreiform movements was initiated with plan to increase tetrabenazine by $12.5 \mathrm{mg}$ increments weekly up to a goal dose of 25mg twice daily. Our patient had a McLeod blood group phenotype sent given the potential risk of future severe transfusion reactions. Unfortunately, the sample for our patient 
was inadequate for interpretation. Thus, his current diagnosis remains neuroacanthocytosis of unspecified subtype.

\section{$\underline{\text { References }}$}

1. Termsarasab P. Chorea. Continuum (Minneap Minn). 2019;25(4):1001-1035.

2. Singer HS. Tics and Tourette Syndrome. Continuum (Minneap Minn). 2019;25(4):936-958.

3. Pfeiffer RF. Wilson Disease. Continuum (Minneap Minn). 2016;22(4):1246-1261.

4. Velayos Baeza A, Dobson-Stone C, Rampoldi L, et al. Chorea-Acanthocytosis. 2002 Jun 14 [Updated 2019 Apr 18]. In: Adam MP, Ardinger HH, Pagon RA, et al., editors. GeneReviews ${ }^{\circledR}$ [Internet]. Seattle (WA): University of Washington, Seattle; 1993-2020

5. Jung HH, Danek A, Walker RH. Neuroacanthocytosis syndromes. Orphanet J Rare Dis. 2011;6:68.

6. Peikert K, Danek A, Hermann A. Current state of knowledge in Chorea-Acanthocytosis as core Neuroacanthocytosis syndrome. European Journal of Medical Genetics. 2018;61:699-705.

7. Fahn S, Jankovic J. Principles and Practice of Movement Disorders. 2007. Churchill Livingston Elsevier. p.23.

8. Schneider S, Lang A, Moro E, Bader B, Danek A, Bhatia K. Characteristic head drops and axial extension in advanced chorea-acanthocytosis. Mov Disord. 2010;25:1487-91.

9. Danek A, Walker R. Neuroacanthocytosis. Curr Opin Neurol. 2005;18:386-392. 
Figure 1: MRI brain T-2 weighted sequence demonstrating bilateral atrophy of the caudate nucleus.

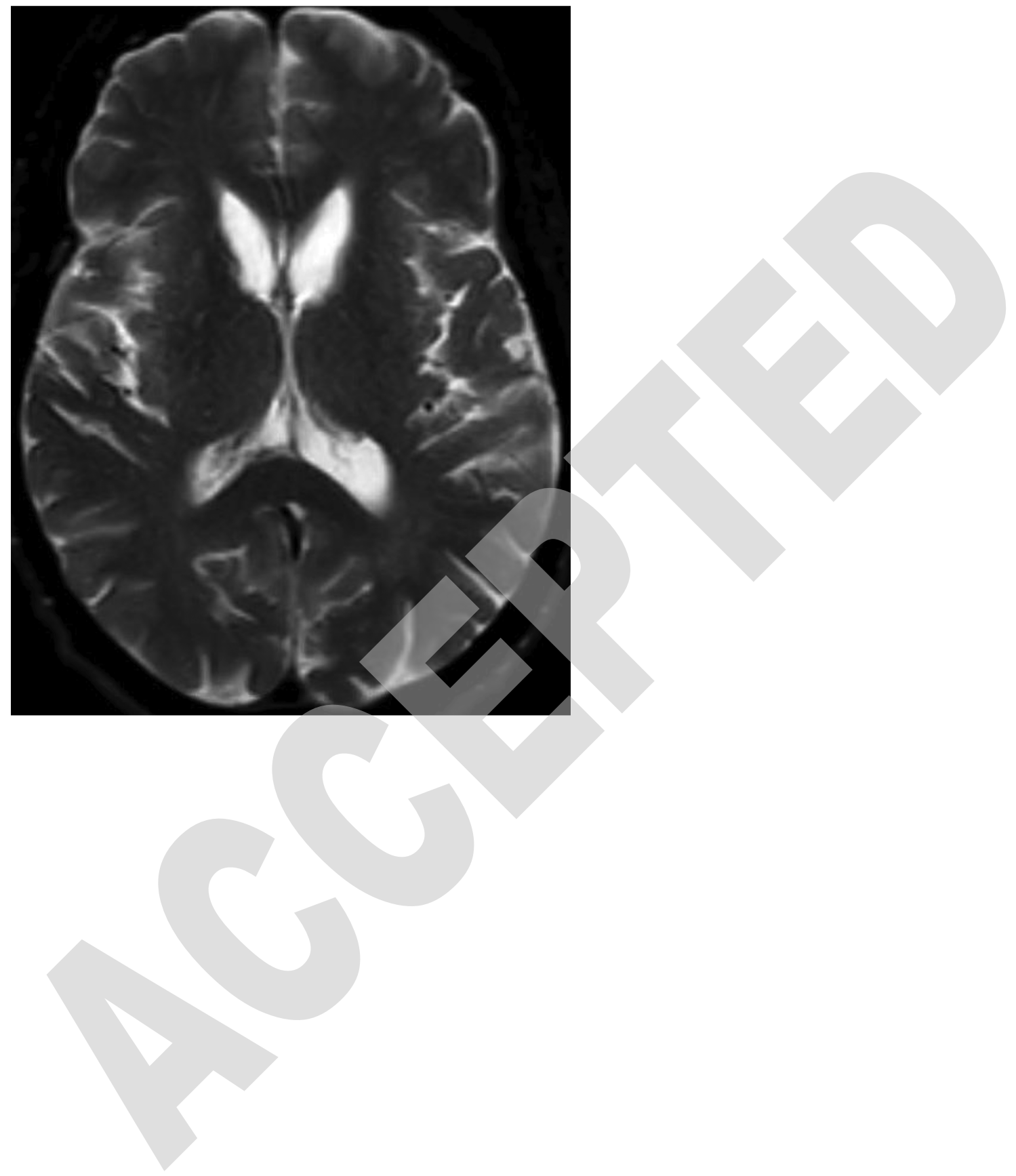




\section{Neurology}

\section{Clinical Reasoning: A 55-Year-Old Man With Odd Behavior and Abnormal Movements}

Paul McIntosh and Burton Scott

Neurology published online August 16, 2021

DOI 10.1212/WNL.0000000000012663

\section{This information is current as of August 16, 2021}

Updated Information \&

Services

Subspecialty Collections

Permissions \& Licensing

Reprints including high resolution figures, can be found at:

http://n.neurology.org/content/early/2021/08/16/WNL.0000000000012663. citation.full

This article, along with others on similar topics, appears in the following collection(s):

All Movement Disorders

http://n.neurology.org/cgi/collection/all_movement_disorders

Chorea

http://n.neurology.org/cgi/collection/chorea

Huntington's disease

http://n.neurology.org/cgi/collection/huntingtons_disease

Information about reproducing this article in parts (figures,tables) or in its entirety can be found online at:

http://www.neurology.org/about/about_the_journal\#permissions

Information about ordering reprints can be found online:

http://n.neurology.org/subscribers/advertise

Neurology ${ }^{\circledR}$ is the official journal of the American Academy of Neurology. Published continuously since 1951, it is now a weekly with 48 issues per year. Copyright (C) 2021 American Academy of Neurology. All rights reserved. Print ISSN: 0028-3878. Online ISSN: 1526-632X.

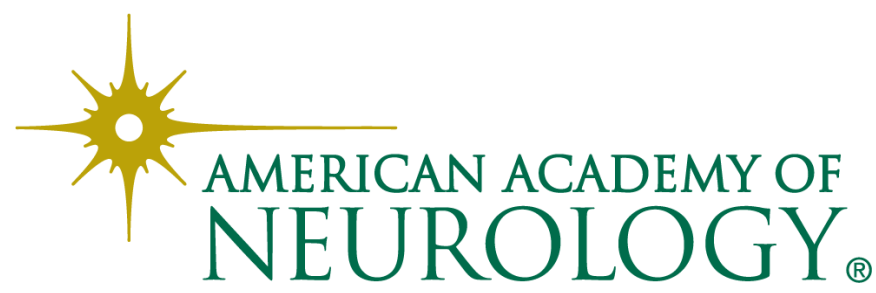

